UCRL-JC-122839

PREPRINT

CONF. $960543 \ldots 7$

\title{
A Tangentially Viewing Visible TV System for the DIII-D Divertor
}

\author{
M.E. Fenstermacher, W.H. Meyer, R.D. Wood, D.G. Nilson, R. Ellis, \\ N.H. Brooks
}

This paper was prepared for submittal to the 11th Topical Conference on High-Temperature Plasma Diagnostics Monterey Conference Center May 12-16, 1996

\section{February 1996}

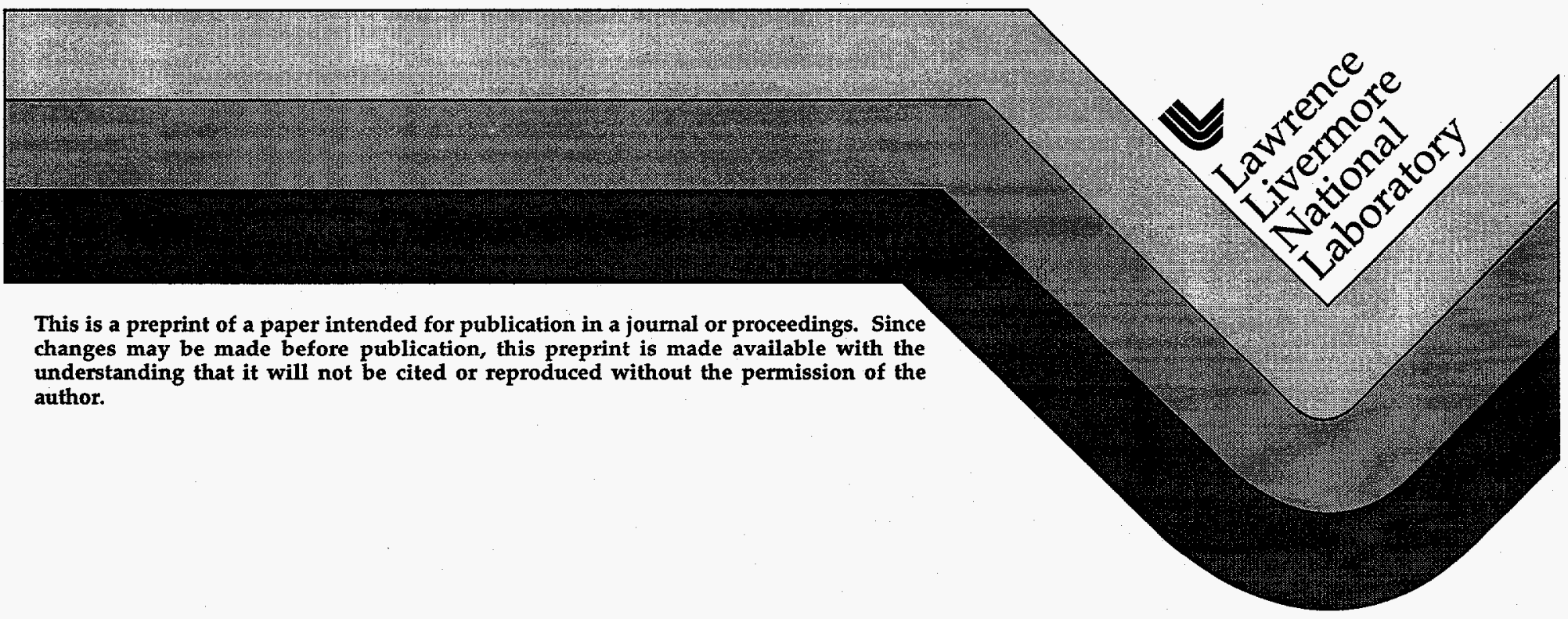




\section{DISCLAMMER}

This document was prepared as an account of work sponsored by an agency of the United States Government. Neither the United States Government nor the University of California nor any of their employees, makes any warranty, express or implied, or assumes any legal liability or responsibility for the accuracy, completeness, or usefulness of any information, apparatus, product, or process disclosed, or represents that its use would not infringe privately owned rights. Reference herein to any specific commercial product, process, or service by trade name, trademark, manufacturer, or otherwise, does not necessarily constitute or imply its endorsement, recommendation, or favoring by the United States Government or the University of California. The views and opinions of authors expressed herein do not necessarily state or reflect those of the United States Government or the University of California, and shall not be used for advertising or product endorsement purposes. 


\title{
A Tangentially Viewing Visible TV System for the DIII-D Divertor
}

\author{
M.E. Fenstermacher, a) W.H. Meyer, R.D. Wood, D.G. Nilson, \\ R. Ellis
}

Lawrence Livermore National Laboratory, Livermore, California

N. H. Brooks

General Atomics, P.O. Box 85608, San Diego, California 92186-9784

A video camera system has been installed on the DIII-D tokamak for 2-D spatial studies of line emission in the lower divertor region. The system views the divertor tangentially from an outer port at approximately the height of the X-point. At the tangency plane the entire divertor from inner wall to outside the DIII-D bias ring is viewed with spatial resolution of approximately $1 \mathrm{~cm}$. The image contains information from approximately 90 degrees of toroidal angle. In a recent upgrade, remotely controllable filter changers were added which have produced images from nominally identical shots using a series of spectral lines. Software was developed to calculate the response function matrix using distributed computing techniques and assuming toroidal symmetry. Standard sparse matrix algorithms are then used to invert the 3-D images onto a poloidal plane. Spatial resolution of the inverted images is $2 \mathrm{~cm}$; higher resolution simply increases the size of the response function matrix. Initial results from a series of experiments with multiple identical shots show that the emission from $\mathrm{CII}$ and CIII, which appears along the inner scrape-off layer above and below the X-point during ELMing 
$\mathrm{H}$-mode, moves outward and becomes localized near the $\mathrm{X}$-point in Partially Detached Divertor (PDD) operation. 


\section{INTRODUCTION}

Extrapolation of results from present diverted tokamaks to the International Thermonuclear Experimental Reactor (ITER) and full scale power producing reactors indicates that the peak heat flux on the divertor target plates will exceed anticipated materials capabilities by more than a factor of 10. A promising technique for reducing the target heat flux is by radiating power in the divertor plasma upstream of the plates thereby spreading the heat load over a much larger area. Experiments are being conducted on DIII-D and many other tokamaks to quantify this "radiative divertor" operating regimel. Spectroscopic measurements along chords indicate that both hydrogenic and impurity radiation play significant roles in radiative divertor operation. These line integrated measurements must be combined with independent data on the spatial extent of the various radiating species in order to quantify the contributions to the total radiated power and to develop predictive capabilities for extending the results to future larger scale devices.

This paper describes a video system for detection of visible line emission which is installed on the DIII-D tokamak. The diagnostic collects image data onto video tape from the lower divertor region (Fig 1). The view of the diagnostic is oriented tangential to the plasma near the position of the $\mathrm{X}$-point for typical lower single null plasmas and spans the entire divertor region from the center post to outside the ADP ring and baffle structure (see Section II). The diagnostic has a long field of view so image data includes information from approximately 90 degrees of toroidal angle. This information is unfolded, assuming 
toroidal symmetry of the radiation, to 2-D distributions in a poloidal plane (see Section III).

\section{HARDWARE}

The tangential view of the diagnostic is obtained by mounting a stainless steel (718) mirror in the divertor baffle at $\mathrm{R}=1.908 \mathrm{~m}, \mathrm{Z}=$ $-1.11 \mathrm{~m}$ (see Fig. 1). This position is recessed in the baffle so that the mirror top is below the level of the surrounding baffle carbon tiles. Special fractional height tiles are used along the line of sight of the diagnostic to improve the view of the divertor floor closest to the mirror. The remaining full thickness baffle tiles are sufficient to protect the mirror from excessive heat flux during normal divertor operation. However, DIII-D operates a wide variety of plasma experiments some of which produce much higher heat flux in the outer regions of the plasma and significantly different pitch angles of the field lines at the mirror position than the nominal case. To protect the mirror as much as possible, close fitting carbon blocks were designed and installed adjacent to the mirror (Fig. 2). Subsequent inspections after months of operation show that substantial heat flux is incident on these carbon blocks and little evidence of heating of the mirror is observed.

The mirror is mounted on a re-entrant tube which is installed on the port below the baffle plate. The canted mirror is mounted on the end of the tube and a vacume window is mounted below it allowing the collected light to be passed out of the vacume region. A rotatable vacume feedthrough is also mounted through the end of the re-entrant tube allowing a carbon shutter assembly to be positioned over the vacume 
window between shots. Various techniques have been used to bring the light out of the tokamak. Initially a rigid borescope (Olympus F-100-107$000-55,107 \mathrm{~cm}$ long, acceptance angle $\pm 27.5^{\circ}, f / 20$ ) was used for measurements of the strong $\mathrm{D}_{\alpha}$ line emission from the divertor. In a recent upgrade, this high loss component was replaced with a fast minilens and fiber image guide assembly (Schott IG-1635 and Schott IG-154, $274 \mathrm{~cm}$ long respectively, see Fig. 3). The mini-lens is approximately $f / 1.1$ and $3 \mathrm{~mm}$ diameter mounted in a $10 \mathrm{~mm}$ diameter housing, and has an acceptance angle of \pm 20 degrees. The fiber image guide is a square bundle, $4 \mathrm{~mm}$ on a side, ${ }^{2}$ constructed of $10 \mu \mathrm{m}$ fibers of standard commercial glass. The fiberguide numerical aperature is $\mathrm{NA}=0.56(f / 0.9)$.

This combination was chosen primarily because it fit into the preexisting design of the re-entrant tube and it provided a substantial improvement in the light throughput of the system for a reasonable cost. The major disadvantage of the system is that the commercial glass fibers are susceptible to neutron damage (browning). Initial transmission fraction for the $3 \mathrm{~m}$ guide in this system is $27 \%$ at $465 \mathrm{~nm}$ (CIII), $35 \%$ at $514 \mathrm{~nm}(\mathrm{CII})$, and $37 \%$ at $656 \mathrm{~nm}\left(\mathrm{D}_{\alpha}\right)$. On DIII-D the neutron fluence is sufficient to substantially reduce the transmission of the fiber imageguide at the short wavelengths in three months of continuous operation. The system features a modular design and at present the imageguide is removed from the machine hall except on days for which the diagnostic is critical to the experiment. The transmission decrease as a function of neutron fluence and wavelength is documented each time the imageguide is removed from the machine hall. This allows a single imageguide to give acceptable performance for selected experiments during about a year of DIII-D operation. 
The design includes a water cooled jacket surrounding the imageguide inside the re-entrant tube so that it can remain on the tokamak during high temperature baking. Maximum temperature on the imageguide is $120^{\circ} \mathrm{C}\left(250^{\circ} \mathrm{F}\right)$. Parts of the DIII-D vacume vessel are baked to $350^{\circ} \mathrm{C}$; the re-entrant tube typically reaches approximately $140^{\circ} \mathrm{C}$ during high temperature baking. At the contact point between the cooling jacket assembly and the re-entrant tube, the copper jacket and the imageguide are separated from the re-entrant tube by a VESPEL spacer. Thermocouple measurements during vacume vessel bakes to $350^{\circ} \mathrm{C}$ indicate that, with active cooling, a temperature differential of greater than $75^{\circ} \mathrm{C}$ is sustained by approximately $0.4 \mathrm{~cm}$ of VESPEL. This is sufficient to keep the imageguide below the damage threshhold temperature.

The distal end of the imageguide is connected to a $25 \mathrm{~mm} f / 1.3$ lens (Computar V2513) with a custom made coupler made of VESPEL which provides a high voltage break $(<5 \mathrm{kV})$ between the tokamak and the remainder of the optics. Coupling to the camera is done with a $50 \mathrm{~mm}$ $f / 1.3$ lens (Computar V5013). The lenses are focussed at infinity and face each other at a separation of $11.4 \mathrm{~cm}$ (4.5 in.). This provides a nearly collimated light beam into which are inserted $25 \mathrm{~mm}$ diameter neutral density and interference filters. The filters are mounted in remotely controllable filter changers (Saguaro Scientific Corporation, Phoenix AZ) which are connected via standard cables to selection units in the voltage isolated section of the DIII-D control room. Each changer holds six filters; changes are made between tokamak shots.

A Charge Induction Device (CID) camera is used (CIDTEK 3710). Unlike typical CCD cameras, CID imagers are very insensitive to neutron 
damage. The camera is sensitive to magnetic fields however; it was necessary to mount it greater than $2.0 \mathrm{~m}$ from the tokamak to obtain continuous operation in all tokamak discharges. Image data is relayed to the control room via fiberoptic links and recorded at standard video rate ( $30 \mathrm{~Hz}, \mathrm{RS}-330$ ) on high quality VHS tape for subsequent digitization and analysis. Real time monitoring and post shot playback of the tape provides a very useful qualitative view of the evolution of the divertor plasma during the shot.

Spatial resolution of the images is approximately $1 \mathrm{~cm}$ at the tangency plane. Side lab tests showed that the system can resolve two horizontal lines, $0.5 \mathrm{~cm}$ apart, at the tangency plane. The camera frames at standard video rates (30 frames/s) and operates in 2:1 interlaced mode. Full frames $(512 \times 512)$ pixels have $32 \mathrm{~ms}$ resolution. Single fields ( 256 vertical lines) have $16 \mathrm{~ms}$ resolution with half the vertical spatial resolution.

In situ spatial and intensity calibrations are performed during vents of the DIII-D vacuum vessel. A spatial calibration grid (plate with holes which are backlit) is oriented along a radial vector and placed at three toroidal positions in the tokamak. Parameters in the image analysis software (e.g. effective detector position and line of sight) are adjusted to obtain consistency between simulated images of the calibration grid and the measurements. Absolute intensity calibration data is obtained using a calibrated integrating sphere light source (Optronics Laboratory, Labsphere 6000). Camera linearity is checked with the calibrated wavelength spectrum from the source using neutral density filters. Linearity of the 8-bit camera is observed up to approximately 150 counts. The intensity calibration is done at selected wavelengths using the interference filters that will be used in the plasma experiments. The 
throughput of the system for many of the filters is too low to allow calibration with the source at its calibrated intensity. The maximum intensity available from the source is used and the wavelength spectrum at that intensity can in principle be extrapolated from the calibration data provided by the manufacturer. Work to obtain this calibration is in progress.

\section{IMAGE ANALYSIS SOFTWARE}

The viewing geometry of this system produces pixel data which is integrated along a long tangential line of sight in the divertor. General 3-D tomographic reconstruction software, originally developed to produce 2-D images of radiated power from chordal bolometer measurements ${ }^{2}$ has been modified to produce 2-D images from the pixel data generated by this camera. Each pixel is treated as a chordal measurement, the imaging geometry is taken into account by a transformation matrix, $M$, and the 2-D distribution in a poloidal plane, assuming toroidal symmetry, is obtained by solving the matrix equation

$$
\mathrm{A} M=\mathrm{B}
$$

using least squares regression techniques. Here $\mathrm{A}$ is the desired 2-D image data, and $\mathrm{B}$ is the raw data including 3-D (toroidal) effects.

Digitized raw camera data is produced in the form of a $512 \times 512$ matrix and reshaped to vector $B$ above $(1 \times 262144)$. For $1 \mathrm{~cm}$ resolution in the real space geometry of the DIII-D divertor, the 2-D image in the poloidal plane must be $100 \times 60$ (vector $A$ dimension $1 \times 6000$ ). This produces a prohibitively large inversion matrix M (1.57 G-samples, or 
6.3 GB). Even storage of the non-zero elements of $M$ and their indecies (approximately $10 \%$ of the full matrix for the Tangential TV view) is an intractably large amount of data. At present the raw data is resampled to $128 \times 128$ and the inversion image is generated with $2 \mathrm{~cm}$ resolution $(50 \times 30)$. The non-zero elements of this matrix and location indices require $9 \mathrm{MB}$ of storage.

A 3-D integral must be evaluated for each matrix element of $M$. These calculations are all independent; distributed computing techniques were used to perform these calculations. Unused cycles during the early morning hours were used on approximately 45 workstations across the U.S.

The spatial calibration of the images is subject to translational and rotational variation due primarily to the repeated removal and reinstallation of the imageguide. An $\mathrm{X}$ application has been written which allows the user to correct for these variations by aligning the images with fiducials generated either from equilibrium calculations (using the EFITD code) $)^{3}$ or from image data in which known structures in the tokamak are illuminated (usually at the end of the shot during the rampdown). This alignment step is performed for each shot, and all images generated from different timeslices for that shot use the same alignment correction.

\section{SAMPLE RESULTS}

Figure 4 shows an example of the raw 3-D image data, the 2-D inversion on a poloidal plane assuming toroidal symmetry, and a reconstructed 3-D image using the 2-D solution integrated toroidally as the camera would view it. Comparison of the 3-D images provides a 
check on the effect of resampling the original data by a factor of 4 . Typically smoothing is observed but the major features of the image are retained.

Figure 5 gives an example of the kind of shift in the emission distribution that has been observed when the divertor plasma makes a transition from ELMing $\mathrm{H}$-mode operation to the radiative divertor mode. The visible emission from CIII is shown in the inner scrapeoff layer region during the ELMing $\mathrm{H}$-mode phase. When a radiative divertor is formed by injection of deuterium gas into the region below the $\mathrm{X}$-point, the CIII emission shifts outward and coelesces near the $\mathrm{X}$-point. This diagnostic, in combination with others which measure plasma and radiation properties in the divertor, has been used in sequences of identical shots to determine the spatial distribution of several of the important radiating species during radiative divertor operation. Work to benchmark 2-D computer models of the divertor plasma and impurities against this data is underway.

\section{CONCLUSIONS}

A tangentially viewing video system is installed on the DIII-D tokamak for observations of visible line emission from the lower divertor region. The optics assembly includes remotely controllable filter changers so that data from multiple emission lines can be obtained from nominally identical, sequential tokamak shots. Software is avaliable to reconstruct from the 3-D image data, a 2-D projection on a poloidal plane assuming toroidal symmetry of the radiation. Data is obtained with $32 \mathrm{~ms}$ temporal and $1 \mathrm{~cm}$ spatial resolution $(16 \mathrm{~ms}$ temporal with $1 \mathrm{~cm}$ 
horizontal and $2 \mathrm{~cm}$ vertical resolution is also available). In vessel spatial and intensity calibration data exists so that in principle absolute calibrations should be possible.

\section{ACKNOWLEDGEMENT}

Work supported by the Department of Energy under Contract Nos. W-7405-ENG-48 and DE-AC03-89ER51114. 


\section{REFERENCES}

a)Present address: General Atomics, P.O. Box 85608, San Diego, California 92186-9784.

1S.L. Allen, "Recent DIII-D Divertor Research," presented at 22nd EPS Conference on Controlled Fusion and Plasma Physics, Bournemouth, United Kingdom, 1995.

${ }^{2}$ A.W. Leonard, W.H. Meyer, B. Geer, D.M. Behne, and D.N. Hill, "2D Tomography with Bolometry in DIII-D," Rev. Sc.i Instrum., Vol. 66, pp. 1201, 1995.

${ }^{3}$ L.L. Lao, H. St. John, R.D. Stambaugh, A.G. Kellman, and W. Pfeiffer, Nucl. Fusion, Vol. 25, pp. 1611, 1985. 


\section{Figure Captions}

Fig. 1. Cross section of the lower divertor region of DIII-D showing the location of the TTV re-entrant tube. The viewing region of the diagnostic is roughly centered about the x-point of a typical lower single null equilibrium.

Fig. 2. Schematic of the TTV re-entrant tube head showing the mirror, carbon shutter assembly and guard carbon blocks. Dashed lines show that the last clear field lines do not intersect the top of the mirror for typical equilibria.

Fig. 3. Schematic of the entire TTV diagnostic showing the re-entrant tube assemply, the fiber imageguide, optics, remotely controllable filter changers and the CID camera.

Fig. 4. Images of CIII emission showing a) the 3-D raw data from the camera, b) a reconstructed 3-D image using the 2-D solution integrated toroidally assuming symmetry, and 3) the 2-D solution overlaid on the equilibrium contours and vacume vessel outline.

Fig. 5. Images of CIII emission from a DIII-D radiative divertor discharge a) during ELMing H-mode before injection of deuterium gas and b) in Partially Detached Divertor operation after gas injection. 


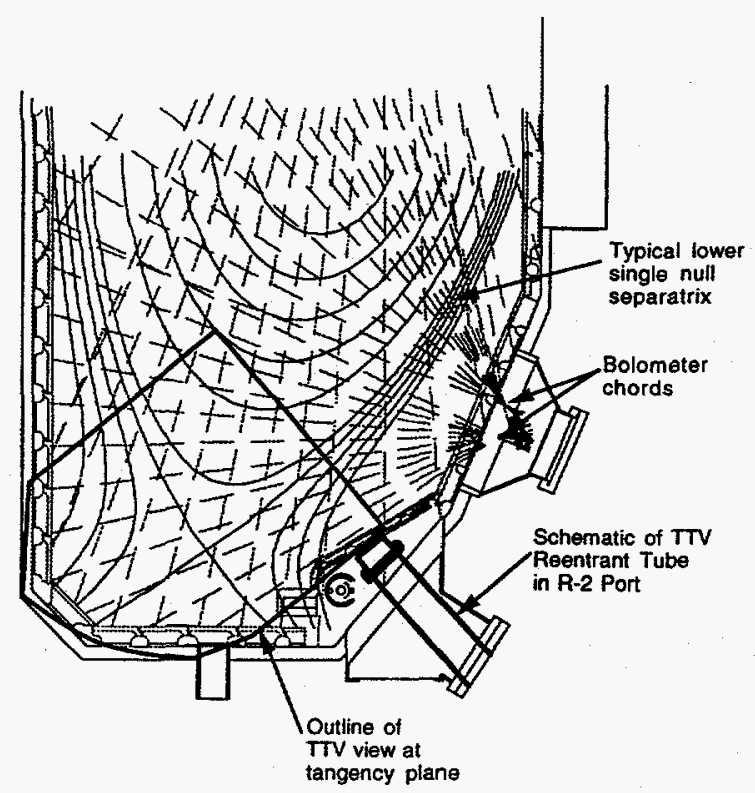

fig 


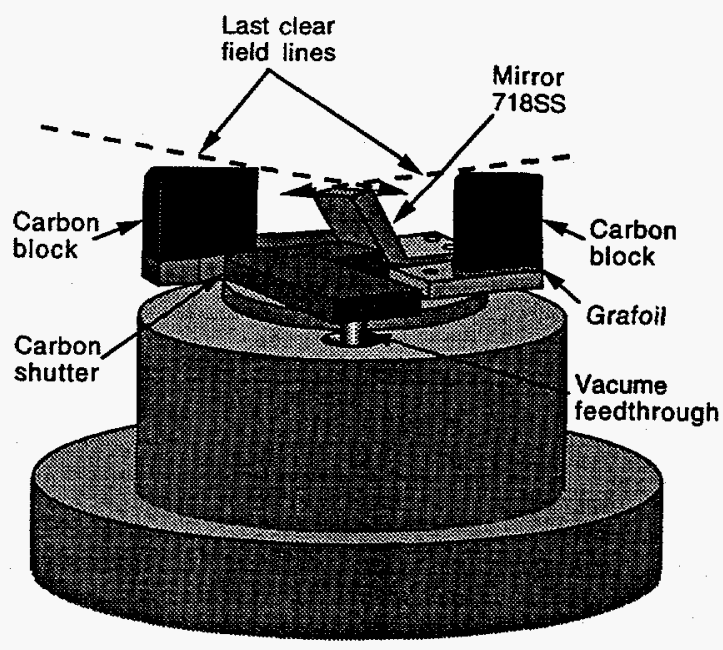

$F / G 2$. 


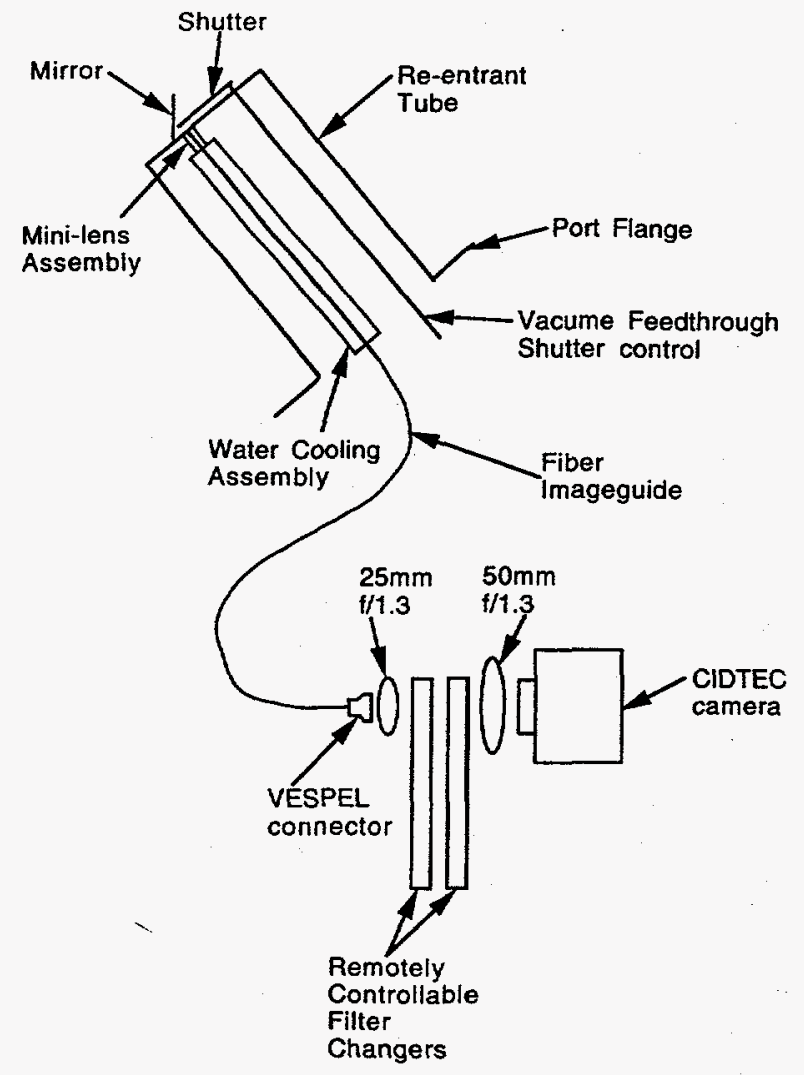

$F / 6.3$ 
Raw Image Data from Camera

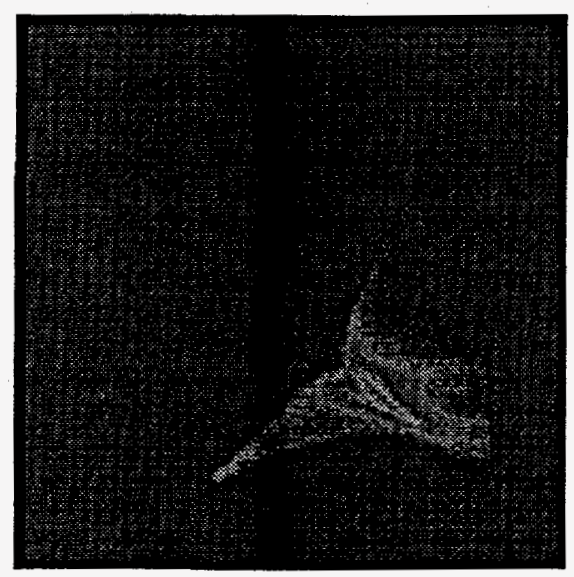

(a)

Virtual Camera Image of Solution

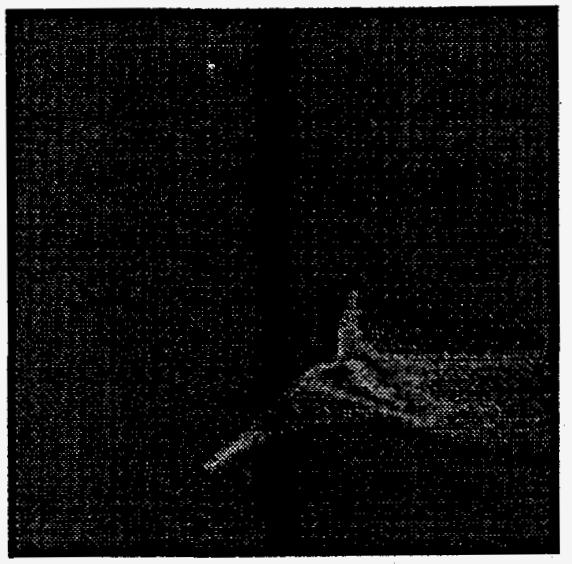

(b)

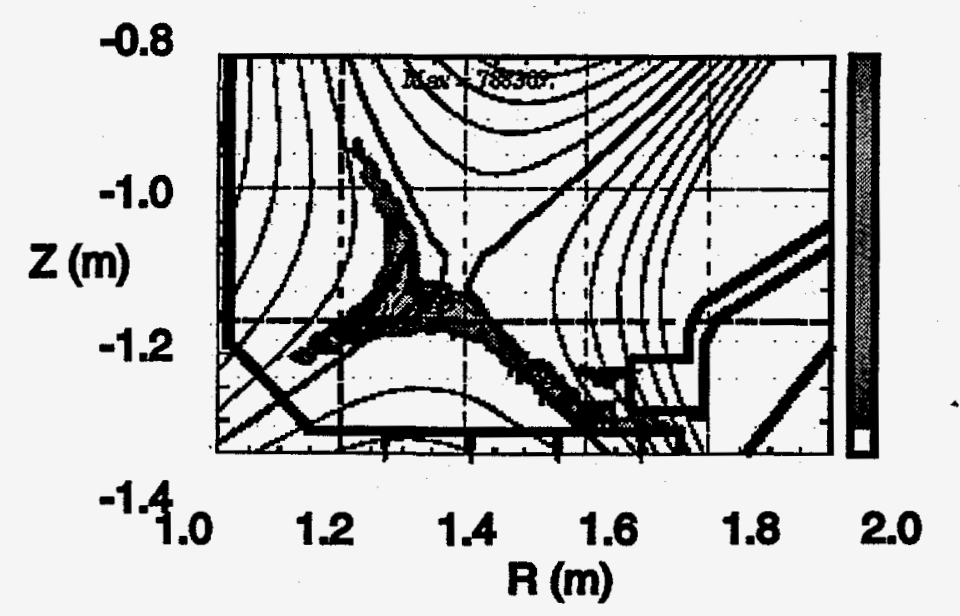

(c) 


$$
\text { 䛑 }
$$


Technical Information Department - Lawrence Livermore National Laboratory University of California - Livermore, California 94551

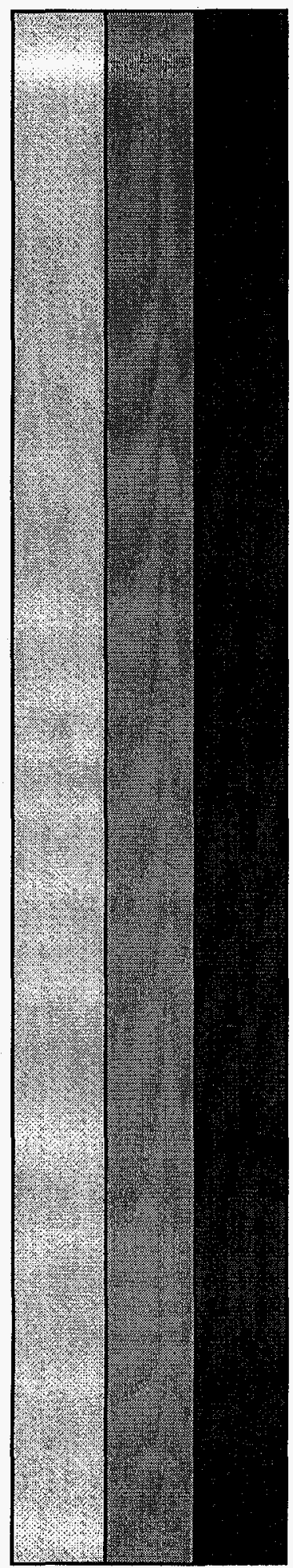

